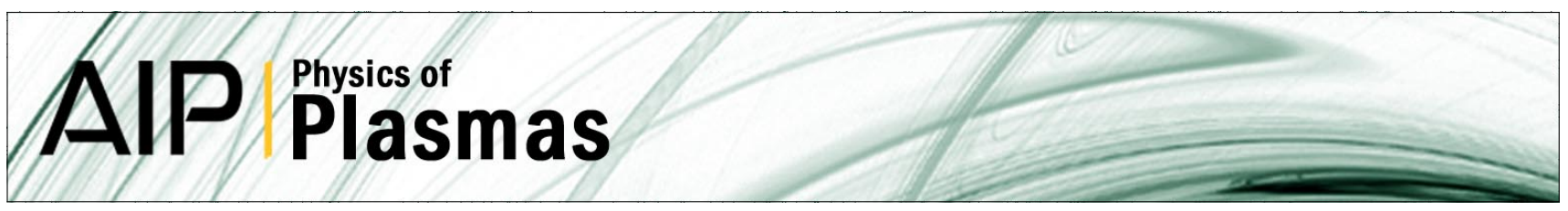

\title{
Potential of a plasma bound between two biased walls
}

J. Loizu, J. Dominski, P. Ricci, and C. Theiler

Citation: Phys. Plasmas 19, 083507 (2012); doi: 10.1063/1.4745863

View online: http://dx.doi.org/10.1063/1.4745863

View Table of Contents: http://pop.aip.org/resource/1/PHPAEN/v19/i8

Published by the American Institute of Physics.

\section{Related Articles}

Efficient proton beam generation from a foam-carbon foil target using an intense circularly polarized laser Phys. Plasmas 19, 083107 (2012)

Parallel transport of long mean-free-path plasmas along open magnetic field lines: Plasma profile variation Phys. Plasmas 19, 082310 (2012)

Oscillating plasma bubbles. IV. Grids, geometry, and gradients

Phys. Plasmas 19, 082108 (2012)

Kinetic Alfven double layer formed by electron viscosity

Phys. Plasmas 19, 072117 (2012)

Physics of the intermediate layer between a plasma and a collisionless sheath and mathematical meaning of the Bohm criterion

Phys. Plasmas 19, 073514 (2012)

\section{Additional information on Phys. Plasmas}

Journal Homepage: http://pop.aip.org/

Journal Information: http://pop.aip.org/about/about_the_journal

Top downloads: http://pop.aip.org/features/most_downloaded

Information for Authors: http://pop.aip.org/authors

\section{ADVERTISEMENT}

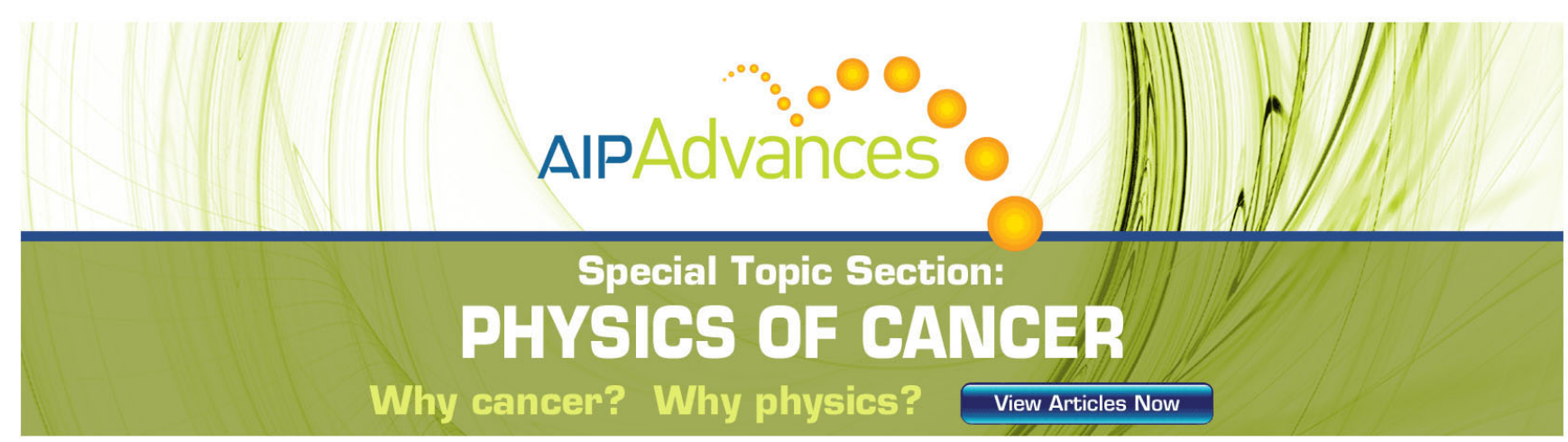




\title{
Potential of a plasma bound between two biased walls
}

\author{
J. Loizu, J. Dominski, P. Ricci, and C. Theiler \\ Centre de Recherches en Physique des Plasmas-École Polytechnique Fédérale de Lausanne, \\ Association EURATOM-Confédération Suisse, CH-1015 Lausanne, Switzerland
}

(Received 25 May 2012; accepted 25 July 2012; published online 17 August 2012)

\begin{abstract}
An analytical study is presented for an one-dimensional, steady-state plasma bound between two perfectly absorbing walls that are biased with respect to each other. Starting from a description of the plasma sheaths formed at both walls, an expression relating the bulk plasma potential to the wall currents is derived, showing that the plasma potential undergoes an abrupt transition when currents cross a critical value. This result is confirmed by numerical simulations performed with a particle-in-cell code. [http://dx.doi.org/10.1063/1.4745863]
\end{abstract}

\section{INTRODUCTION}

At the edge of magnetically confined fusion plasmas, at the interface between spacecraft and space plasmas, in the fabrication of semiconductor devices, and wherever a plasma interacts with a solid surface, the plasma-wall transition is characterized by the presence of a non-neutral sheath, which extends over a few Debye lengths. The existence of the sheath ensures that quasi-neutrality is maintained in the plasma bulk by a strong electric field, typically leading to no net current to the walls.

A more complicated situation is present when a region of the wall in contact with the plasma is electrically biased with respect to the rest of the wall. Time-independent biasing is used in plasma experiments for different purposes, namely for the measure of the ion and electron temperatures with electrostatic probes, ${ }^{2,3}$ in plasma thrusters for space propulsion, ${ }^{4}$ to study the effect of shear flow on turbulence, ${ }^{6-9}$ for the study of dust particles, ${ }^{5}$ and for the control of turbulence in magnetic fusion devices. ${ }^{10-12} \mathrm{~A}$ bias may induce local perturbations of the plasma potential. Electric fields are then produced and can give rise to plasma currents, which may close at the sheath. A commonly shared feature in biasing experiments is that the plasma potential sets its value in between two surface potentials (see, e.g., Ref. 3). While in some relatively simple cases, the underlying physical mechanism has been understood, ${ }^{13}$ the exact general relation between the currents measured at the sheaths, the applied bias, and the resulting potential in the plasma bulk is not well established, and remains to date a challenging general problem of plasma physics. ${ }^{14}$

The goal of the present article is to address this problem in a relatively simple framework, focusing on a onedimensional, steady-state, plasma bound between two perfectly absorbing walls that are biased with respect to each other. In particular, we derive an analytical expression relating the bulk plasma potential with the wall currents, showing that the plasma potential undergoes an abrupt transition when currents cross a critical value. This result is confirmed by numerical simulations performed with a particle-in-cell (PIC) code.

The electrostatic potential established in the plasma bulk depends on the interplay between sheaths driving differ- ent currents to the walls. Typically, sheaths are positive space-charge layers forming a potential barrier, $\eta_{s e}=e\left(\phi_{s e}-\phi_{w}\right) / T_{e}>0$, which prevents most of electrons from flowing out. Here, $\phi_{s e}$ and $\phi_{w}$ are, respectively, the potentials at the sheath edge and at the wall, and $T_{e}$ is the electron temperature. An enormous research effort on these sheaths, called ion sheaths, has been carried out in the past decades (see Ref. 1 for a review). Standard sheath theory shows that the sheath current $I$ is such that $I_{\text {sat }}^{e l}<I<I_{\text {sat }}^{i o n}$ for $\eta_{s e}>0$, where $I_{\text {sat }}^{\text {ion }}=e n_{s e} c_{s}>0$ is the ion saturation current and $I_{\text {sat }}^{e l}=-e n_{\text {se }} \sqrt{2 / \pi} v_{\text {the }}<0$ is the electron saturation current, with $n_{s e}$ the sheath edge density, $c_{s}=\sqrt{T_{e} / m_{i}}$ and $v_{\text {the }}=\sqrt{T_{e} / m_{e}}$. When a strong positive bias is locally applied with a probe or at the wall confining the plasma, the formation of negative space-charge sheaths or electron sheaths is observed. ${ }^{3,10,13}$ In the case of the electron sheath, a potential barrier $\eta_{s e}<0$ accelerates electrons and prevents most of ions from arriving at the wall (Figure 1). As a matter of fact, biasing experiments often show that the plasma is bound between an ion and an electron sheath. This is the plasma scenario that we consider in the present paper, which starts with the analysis of the ion and electron sheaths, shedding new light on their properties. These results are then used to describe the interplay between the two sheaths and their effect on the bulk plasma potential.

The article is organized as follows. In Sec. II, we analyse the plasma dynamics in both ion and electron sheaths, deriving the ion and electron velocities at the sheath edge. These results are used in Sec. III to describe a plasma bound between two biased walls. We derive an analytical expression relating the plasma potential, the wall currents, and the applied bias. We find that the plasma potential undergoes an abrupt transition when currents cross a given critical value. In Sec. IV, the analytical predictions are numerically verified by using a PIC code. In the final discussion and conclusion, Sec. V, we discuss the implications of our predictions on biasing experiments.

\section{SHEATHS}

The goal of the present section is to find the ion and electron velocities at the sheath entrance in the case of perfectly absorbing walls. We consider separately the ion and 
(a)

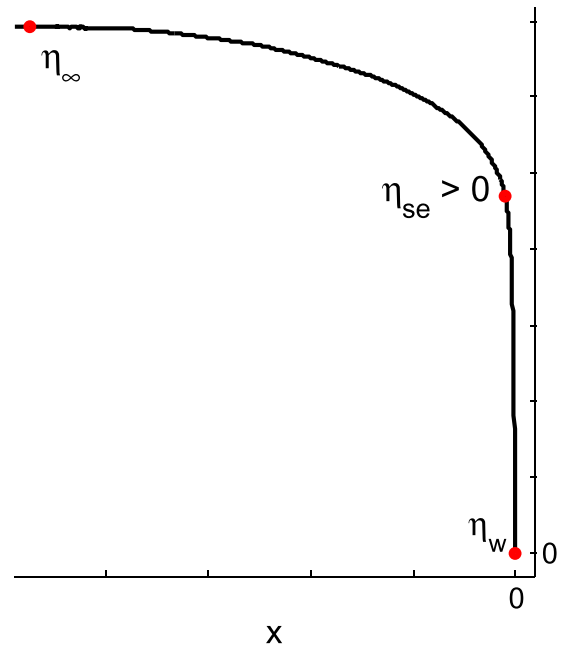

(b)

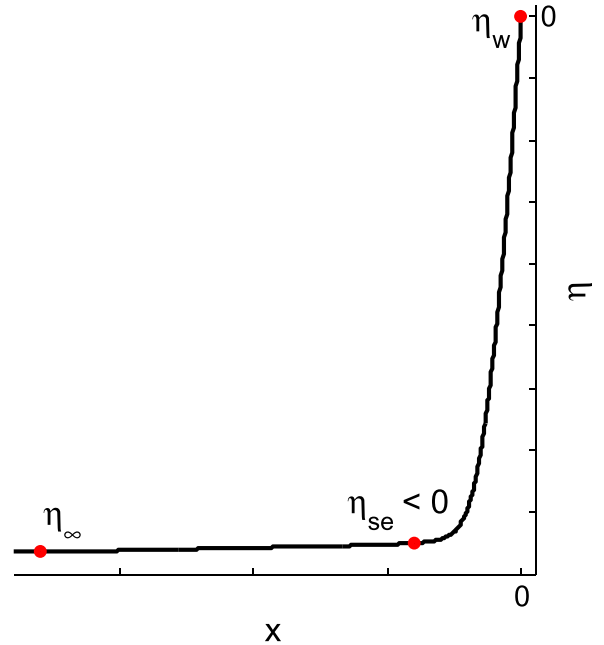

FIG. 1. Example of potential drop $\eta$ as a function of the distance to the wall for an ion sheath (left) and an electron sheath (right). Indicated are the electrostatic potential far from the wall $\left(\eta_{\infty}\right)$, at the sheath edge $\left(\eta_{s e}\right)$, and at the wall $\left(\eta_{w}=0\right)$. Plots are obtained with the PIC code described in Sec. IV. the electron sheaths, i.e., $\eta_{s e}>0$ and $\eta_{s e}<0$, respectively. The results presented herein are valid for unmagnetized plasmas as well as for magnetized plasmas when the magnetic field is perpendicular to the walls. We also note that in the following, we consider singly charged ions.

\section{A. Ion sheaths}

Let us first consider a plasma in contact with an absorbing wall in the case of an ion sheath where $\eta_{s e}>0$ (Fig. 1(a)). In this situation, the ion and electron velocities at the sheath edge were recently derived in the limit of cold ions. ${ }^{15}$ We now extend the results to the case of finite ion temperature.

In the presence of a monotonic ion sheath, the electron fluid velocity in the direction normal to the wall is given by

$$
V_{e}=\frac{v_{\text {the }}}{\sqrt{2 \pi} I(\eta)} e^{-\eta}=\frac{c_{s}}{I(\eta)} e^{\Lambda-\eta},
$$

where $\quad c_{s}=\sqrt{T_{e \infty} / m_{i}}, \Lambda=\log \sqrt{\mu / 2 \pi}, \mu=m_{i} / m_{e} \quad$ and $I(\eta)=[1+\operatorname{erf}(\sqrt{\eta})] / 2$. Here, $\eta(x)=e\left(\phi(x)-\phi_{w}\right) / T_{e \infty}$ is the normalized potential relative to the wall such that $\eta(0)=0$ and $T_{e \infty}$ is the electron temperature far from the wall (in the bulk plasma). Equation (1) results from computing the first moment of the electron distribution function, which is a truncated Maxwellian in the proximity of a perfectly absorbing wall. ${ }^{15}$ Notice that the spatial dependence of $V_{e}$ is contained in the potential $\eta$. We now consider the continuity equations for ions and electrons and the momentum equation for ions, which in steady state are

$$
\begin{gathered}
n_{i} \frac{\partial V_{i}}{\partial x}+V_{i} \frac{\partial n_{i}}{\partial x}=S_{p i} \\
n_{e} \frac{\partial V_{e}}{\partial x}+V_{e} \frac{\partial n_{e}}{\partial x}=S_{p e} \\
m_{i} n_{i} V_{i} \frac{\partial V_{i}}{\partial x}=-e n_{i} \frac{\partial \phi}{\partial x}-\frac{\partial}{\partial x}\left(n_{i} T_{i}\right)+S_{m i} .
\end{gathered}
$$

The particle and momentum sources of species $\alpha, S_{p \alpha}$, and $S_{m \alpha}$ are related to the injection of particles, ionization processes, or collisions. System (2) can be reduced to a ma- trix equation. First, the term $\partial_{x} V_{e}$ can be evaluated as $\partial_{x} V_{e}=\partial_{\phi} V_{e} \partial_{x} \phi$; and from Eq. (1), it follows that $\partial_{\phi} V_{e}=-\left(e V_{e} / T_{e \infty}\right)\left[1+e^{-\eta} / 2 \sqrt{\pi \eta} I(\eta)\right]$. Second, the term related to the ion pressure, $\partial_{x}\left(n T_{i}\right)$ can be simplified by assuming that the ion fluid expands (accelerates) adiabatically, namely without heat exchange. This leads to $d\left(n_{i}^{1-\gamma} T_{i}\right) / d t=0$ and thus $\partial_{x}\left(n_{i} T_{i}\right)=\gamma T_{i} \partial_{x} n_{i}$, where the coefficient $\gamma$ is given by the kinetic theory of gases as $\gamma=(\nu+2) / \nu, \nu$ being the number of degrees of freedom of the particles (for one-dimensional flow $\gamma=3$ ). Finally, we note that in the presheath and up to the sheath entrance, quasi-neutrality is preserved and the condition $n_{e}=n_{i}=n$ has to be fulfilled. Therefore, our system of equations can be reduced to a matrix equation $\mathbf{M} \vec{X}=\vec{S}$, where

$$
\vec{X}=\left(\begin{array}{c}
\partial_{x} n \\
\partial_{x} V_{i} \\
\partial_{x} \phi
\end{array}\right), \quad \vec{S}=\left(\begin{array}{c}
S_{p i} \\
S_{p e} \\
S_{m i}
\end{array}\right)
$$

and

$$
\mathbf{M}=\left(\begin{array}{ccc}
V_{i} & n & 0 \\
V_{e} & 0 & n \partial_{\phi} V_{e} \\
\gamma T_{i} & m_{i} n V_{i} & e n
\end{array}\right)
$$

In the presheath region, gradients are typically small and are due to the presence of the plasma source. At the sheath edge, gradients become much larger, i.e., $\left|M_{\alpha \beta} X_{\beta}\right| \gg\left|S_{\alpha}\right|$ for all $\alpha, \beta$ such that $M_{\alpha \beta} \neq 0$. In other words, at the sheath edge, the source terms are much smaller than the other terms in the fluid equations, and System (2) reduces to $\mathbf{M} \vec{X} \simeq 0$. The presence of non-zero gradients imposes $\operatorname{det}(\mathbf{M})=0$, which defines the position of the sheath edge. We note that $\operatorname{det}(\mathbf{M})=0$ is also a valid definition of the sheath edge in the particular case of a source-free system. In this case, $\mathbf{M} \vec{X}=0$ is satisfied everywhere in the presheath and the macroscopic quantities display flat profiles, $\vec{X}=0$, up until the sheath entrance, ${ }^{16}$ where gradients become non zero, therefore still requiring $\operatorname{det}(\mathbf{M})=0$. Hence in all cases, $\operatorname{det}(\mathbf{M})=0$ at the sheath entrance, which gives 


$$
V_{i, s e}=c_{s} \sqrt{\frac{1}{1+\kappa\left(\eta_{s e}\right)}+f_{i}},
$$

where the function $\kappa$ is defined as

$$
\kappa(\eta)=\frac{e^{-\eta}}{2 \sqrt{\pi \eta} I(\eta)}
$$

and represents the kinetic effect of the depleted Maxwellian electron distribution function. ${ }^{15}$ This effect becomes important when $\eta \rightarrow 0$, while it vanishes for $\eta \rightarrow \infty$. Also, we define

$$
f_{\alpha}=\gamma \frac{T_{\alpha, s e}}{T_{e \infty}},
$$

which represents the effect of a finite-temperature fluid of species $\alpha$ expanding adiabatically. We note that $f_{i}$ is related to the presheath density drop. In the case of adiabatic flow, in fact, $T_{i, s e} / T_{i \infty}=\tilde{n}_{s e}^{\gamma-1}$, where $\tilde{n}_{s e}=n_{s e} / n_{\infty}$ is the sheath edge density normalized to the bulk plasma density $n_{\infty}$. Therefore, $f_{i}=\gamma \tau \tilde{n}_{s e}^{\gamma-1}$ where $\tau=T_{i \infty} / T_{e \infty}$. As a consequence, $f_{i} \rightarrow 0$ for $\tau \rightarrow 0$, and thus Eq. (5) reduces to the Bohm criterion, $V_{i}=c_{s}$, in the limits $\eta_{s e} \rightarrow \infty$ and $\tau=0$. Another well-known result is retrieved by considering the limit $\eta_{s e} \rightarrow \infty$ for arbitrary $\tau$, which gives $V_{i}=\sqrt{\left(T_{e \infty}+\gamma T_{i, s e}\right) / m_{i}} .18$

Equations (1) and (5) provide the ion and electron velocities at the entrance of ion sheaths. In particular, one can obtain the so-called floating potential, $\eta_{f}$, for which the flow is ambipolar, by solving $V_{i, s e}=V_{e, s e}$. For $\tau=0$, this gives $\eta_{f} \simeq \Lambda$.

\section{B. Electron sheaths}

Let us now consider the case of an electron sheath, namely $\eta_{s e}<0$ (Fig. 1(b)). In this case, electrons are accelerated through the sheath electric field and are all absorbed, while ions are repelled unless they are sufficiently energetic to overcome the sheath potential barrier, a situation that is reversed with respect to ion sheaths. Therefore, the ion fluid velocity in the direction normal to the wall can be expressed as

$$
V_{i}=\frac{v_{t h i}}{\sqrt{2 \pi} I(|\eta| / \tau)} e^{-|\eta| / \tau}=\frac{c_{s} \sqrt{\tau}}{I(|\eta| / \tau)} e^{-|\eta| / \tau},
$$

where $|\eta(x)| / \tau=e\left(\phi_{w}-\phi(x)\right) / T_{i \infty}$.

In steady state, the continuity equations for electrons and ions, and the momentum equation for electrons are

$$
\begin{gathered}
n_{e} \frac{\partial V_{e}}{\partial x}+V_{e} \frac{\partial n_{e}}{\partial x}=S_{p e} \\
n_{i} \frac{\partial V_{i}}{\partial x}+V_{i} \frac{\partial n_{i}}{\partial x}=S_{p i} \\
m_{e} n_{e} V_{e} \frac{\partial V_{e}}{\partial x}=e n_{e} \frac{\partial \phi}{\partial x}-\frac{\partial}{\partial x}\left(n_{e} T_{e}\right)+S_{m e}
\end{gathered}
$$

As before, the term $\partial_{x} V_{i}$ in System (9) can be evaluated as $\partial_{x} V_{i}=\partial_{\phi} V_{i} \partial_{x} \phi$, and $\partial_{\phi} V_{i}$ can be obtained from Eq. (8).
The quasi-neutrality in the presheath and the adiabaticity of the electron flow lead to a matrix equation $\mathbf{M} \vec{X}=\vec{S}$, where

$$
\begin{gathered}
\vec{X}=\left(\begin{array}{c}
\partial_{x} n \\
\partial_{x} V_{e} \\
\partial_{x} \phi
\end{array}\right), \quad \vec{S}=\left(\begin{array}{c}
S_{p e} \\
S_{p i} \\
S_{m e}
\end{array}\right), \\
\mathbf{M}=\left(\begin{array}{ccc}
V_{e} & n & 0 \\
V_{i} & 0 & n \partial_{\phi} V_{i} \\
\gamma T_{e} & m_{e} n V_{e} & -e n
\end{array}\right) .
\end{gathered}
$$

As for the ion sheath, the conditon $\operatorname{det}(\mathbf{M})=0$ sets the sheath entrance, namely

$$
V_{e, s e}=v_{t h e} \sqrt{\frac{\tau}{1+\kappa\left(\left|\eta_{s e} / \tau\right|\right)}+f_{e}} .
$$

We notice that the electron velocity at the sheath entrance is of the order of the thermal velocity, $v_{\text {the }}$, since all electrons are absorbed. In fact, in the limit $\tau \rightarrow 0$, Eq. (12) gives $V_{e}=\sqrt{\gamma T_{e, s e} / m_{e}} \sim v_{\text {the }}$. The quantity $f_{e}$ can also be related to the presheath density drop by using the assumption of adiabatic flow, i.e., $T_{e, s e} / T_{e \infty}=\tilde{n}_{s e}^{\gamma-1}$, which leads to $f_{e}=\gamma \tilde{n}_{s e}^{\gamma-1}$.

Equations (8) and (12) provide the ion and electron velocities at the entrance of electron sheaths. As a final remark, we mention that in the limit $\eta_{s e} \rightarrow 0$, both electron and ion sheaths disappear and the electron and ion velocities at the wall are given by Eqs. (1) and (8), respectively.

\section{PLASMA BETWEEN TWO BIASED WALLS}

Let us now consider the situation of an one-dimensional, steady-state plasma bound in between two perfectly absorbing walls. Let us call $\phi_{w}^{l}$ and $\phi_{w}^{r}$ the potentials of the left and right walls and denote with $\delta=e\left(\phi_{w}^{r}-\phi_{w}^{l}\right) / T_{e \infty}>0$ the bias applied between the walls. From now on, we use the left wall as the reference for the normalized plasma potential, namely, $\eta(x)=e\left[\phi(x)-\phi_{w}^{l}\right] / T_{e \infty}$. In order to maintain a steady-state, a source replenishes the plasma that is continuously lost at both ends due to the sheath condition. In particular, the plasma source may be non-neutral and currents may be established at the sheaths in order to ensure quasineutrality in the plasma bulk. This situation is very common in biasing experiments, where plasma currents feed the biased region by acting as non-neutral sources. These currents are eventually closed at the sheaths. ${ }^{10}$

Two situations may be observed depending on the electric charge introduced by the source, see Fig. 2. If the plasma source is such that $S_{i} \geq S_{e}$, the plasma potential stays always above the highest wall potential $\phi_{w}^{r}$, and ion sheaths are present on both sides. On the left side, the sheath edge potential is above the floating potential, leading therefore to an ion current, $\left|\Gamma_{i}\right|>\left|\Gamma_{e}\right|$, where $\Gamma_{\alpha}=n_{\alpha, s e} V_{\alpha, s e}$. On the right side, the sheath edge potential is such that the current established maintains the quasi-neutrality. If the source is negatively charged, $S_{i}<S_{e}$, the potential of the plasma bulk approaches $\phi_{w}^{r}$ in order for the sheath to evacuate the excess of electrons (see Fig. 2). If the negative source is strong enough, the plasma potential sets its value below $\phi_{w}^{r}$. In this regime, an 


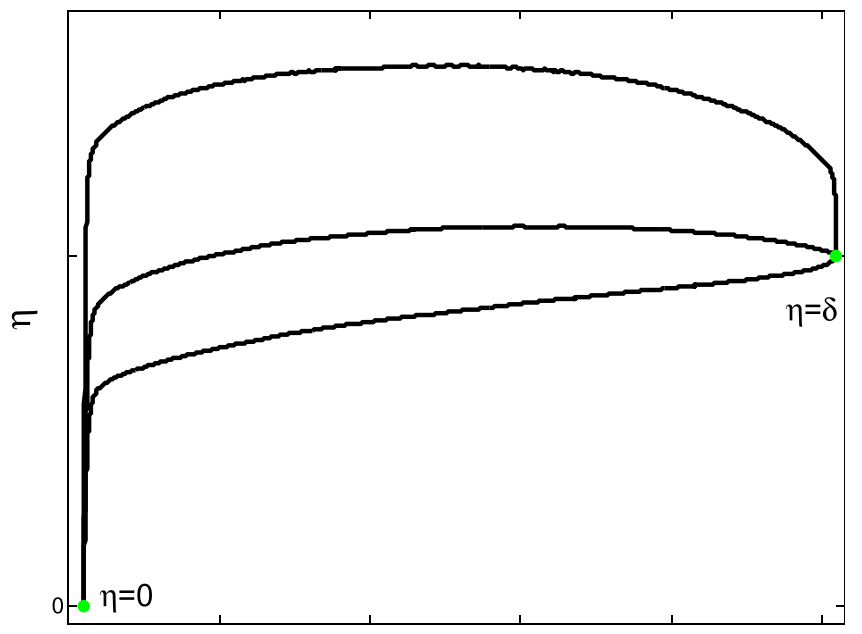

$\mathbf{X}$

FIG. 2. Examples of plasma potential profiles $\eta(x)$ for a bias $\delta=e\left(\phi_{w}^{r}-\phi_{w}^{l}\right) / T_{e \infty}$ applied between the two walls. The top curve is for the case of a neutral plasma source. The middle curve is for a moderately charged negative source $\left(S_{i} \lesssim S_{e}\right)$, while the bottom curve is for a strongly charged negative source $\left(S_{i} \ll S_{e}\right)$. Plots are obtained with the PIC code described in Sec. IV, in the case of $\tau=1$.

ion sheath is established on one wall, while an electron sheath is present on the other wall. This situation is found in many experiments where a positive bias is applied (see, e.g., Refs. 3, 10, and 13). In the following, we focus on this particularly interesting regime. We derive an expression relating the bulk plasma potential, the bias, and the wall currents, by using the results of Sec. II.

We consider the steady-state charge balance of an onedimensional plasma bound between two biased walls, in the presence of a non-neutral plasma source. We define $J_{i e}$ as the ratio between the ion and electron sources,

$$
J_{i e}=\frac{\int_{0}^{L} S_{i} d x}{\int_{0}^{L} S_{e} d x},
$$

where $L$ is the size of the system. From the steady-state continuity equation for ions and electrons, it follows that $J_{i e}$ is also equal to the ratio between the total ion outflux and the total electron outflux,

$$
J_{i e}=\frac{\Gamma_{i w}^{r}-\Gamma_{i w}^{l}}{\Gamma_{e w}^{r}-\Gamma_{e w}^{l}}=\frac{\left|\Gamma_{i w}^{r}\right|+\left|\Gamma_{i w}^{l}\right|}{\left|\Gamma_{e w}^{r}\right|+\left|\Gamma_{e w}^{l}\right|},
$$

where $\Gamma_{\alpha w}^{l}=n_{\alpha w}^{l} V_{\alpha w}^{l}$ and $\Gamma_{\alpha w}^{r}=n_{\alpha w}^{r} V_{\alpha w}^{r}$ are the particle fluxes at the left and right walls. These are all outflowing, i.e., $\Gamma_{\alpha w}^{l}<0$ and $\Gamma_{\alpha w}^{r}>0$.

We assume that inside the sheaths, the effect of $S_{i}(x)$ and $S_{e}(x)$ can be neglected. This can be quantified as $S_{\alpha} \ll n_{s e} \omega_{p i}$, where $\omega_{p i}=\sqrt{e^{2} n_{s e} / \epsilon_{0} m_{i}}$ is the ion plasma frequency. This condition is derived by imposing $S_{\alpha} \ll$ $V_{\alpha} \partial_{x} n$ and taking $V_{\alpha} \sim c_{s}$ and $\partial_{x} \sim 1 / \lambda_{D}$. As a consequence, the particle fluxes are conserved inside the sheaths and thus we can write $\Gamma_{\alpha w}^{l}=\Gamma_{\alpha s e}^{l}$ and similarly for the right wall. The fluxes in Eq. (14) are, therefore, given by the fluxes at each sheath edge, and one can make use of the ion and electron velocities at the sheath entrance derived in Sec. II, i.e., Eqs. (5) and (8) for the ions, and Eqs. (1) and (12) for the electrons, to derive a relation between $J_{i e}$ and the potential in the plasma bulk.

For this purpose, we assume $L$ to be much larger than the sheath length, $L \gg \lambda_{D}$. This allows us to consider the main plasma as infinitely far from both walls, defining the bulk plasma potential as $\eta_{\infty}=\eta(L / 2)$ and its density as $n_{\infty}=n(L / 2)$. We further assume that the normalized bias is large, namely, $\delta=e\left(\phi_{w}^{r}-\phi_{w}^{l}\right) / T_{e \infty} \gg 1$, such that the presheath potential drop can be neglected with respect to the sheath potential drop. It follows that the sheath potential barrier at the left wall is $\eta_{\infty}>0$ (ion sheath) and that at the right wall is $\eta_{\infty}-\delta<0$ (electron sheath). We recall that this situation corresponds to the bottom curve of Fig. 2. Using Eqs. (1), (5), (8), and (12), and $\Gamma_{\alpha w}^{l, r}=\Gamma_{\alpha s e}^{l, r}$, we can write Eq. (14) as

$$
J_{i e}=\frac{1}{\sqrt{\mu}} \frac{\tilde{n}_{l} \sqrt{\frac{1}{1+\kappa\left(\eta_{\infty}\right)}+\gamma \tau \tilde{n}_{l}^{\gamma-1}+\tilde{n}_{r} \frac{\sqrt{\tau} e^{-\left(\delta-\eta_{\infty}\right) / \tau}}{\sqrt{2 \pi} I\left(\left(\delta-\eta_{\infty}\right) / \tau\right)}}}{\tilde{n}_{l} \frac{e^{-\eta_{\infty}}}{\sqrt{2 \pi} I\left(\eta_{\infty}\right)}+\tilde{n}_{r} \sqrt{\frac{\tau}{1+\kappa\left(\left(\delta-\eta_{\infty}\right) / \tau\right)}+\gamma \tilde{n}_{r}^{\gamma-1}}} .
$$

Here, $\tilde{n}_{l}=n_{s e}^{l} / n_{\infty}$ and $\tilde{n}_{r}=n_{s e}^{r} / n_{\infty}$ are the sheath edge densities at the left and right sides normalized to the bulk density, and the identity $c_{s} / v_{\text {the }}=1 / \sqrt{\mu}$ has been used. Equation (15) directly relates $J_{i e}$ to $\eta_{\infty}$, and it is valid for $0<\eta_{\infty}<\delta$, which corresponds to the regime of an ion sheath on one wall and an electron sheath on the other wall. Six parameters modulate the function $J_{i e}\left(\eta_{\infty}\right)$, namely $\mu, \tau, \delta, \gamma, \tilde{n}_{l}$, and $\tilde{n}_{r}$. Figure 3 shows the bulk plasma potential as a function of $J_{i e}$ as given by Eq. (15), for different values of $\tau$ and $\tilde{n}_{l} / \tilde{n}_{r}$.

It is interesting to note that in all cases, there is an abrupt transition of the plasma potential occurring around a critical value of the current ratio $J_{i e}$. This can be explained as follows. When the bulk plasma potential is $\eta_{\infty} \simeq \delta$, the current at the left wall is due to ions entering the sheath at approximately the sound speed, while the current at the right wall is fundamentally due to electrons entering at approximately the thermal speed, thus giving $J_{i e} \simeq 1 / \sqrt{\mu}$. As a matter of fact, the right sheath draws electrons at about the thermal speed regardless of the value of $\eta_{\infty}$, if $\eta_{\infty}<\delta$, since no potential barrier prevents them from being absorbed. On the other hand, the left sheath draws ions at about the sound speed and electrons at a speed that depends on the potential barrier, since $V_{e} \sim c_{s} \exp \left(\Lambda-\eta_{\infty}\right)$, see Eq. (1). This exponential dependence explains why $\eta_{\infty}$ must approach the floating potential $\eta_{f} \simeq \Lambda$ in order for the left sheath to start drawing a significant amount of electron current, therefore changing the value of $J_{i e}$. Thus, for $\eta_{f} \lesssim \eta_{\infty}<\delta$, the left and right sheaths, respectively, draw almost the same ion and electron currents as in the case $\eta_{\infty} \simeq \delta$, thus explaining the sharpness of the transition observed in Fig. 3.

The transition in $\eta_{\infty}$ occurs at a certain current ratio $J_{i e}=J_{t}$, which we identify as the current ratio at which $\eta_{\infty}=\delta / 2$. A general expression for $J_{t}$ can be derived from Eq. (15) by taking simultaneously the limits $\eta_{\infty} \gg 1$ and $\delta-\eta_{\infty} \gg 1$, and it is given by 

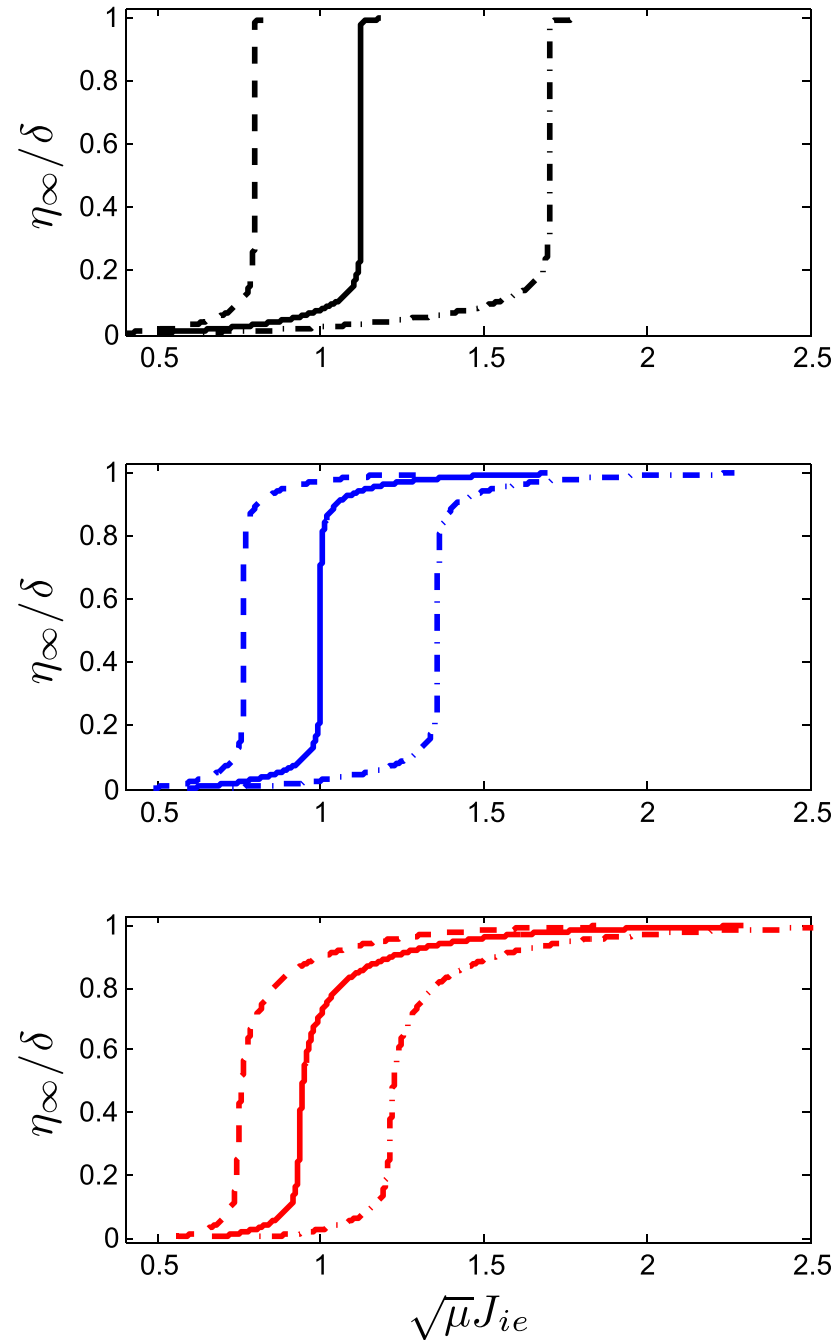

FIG. 3. Normalized main plasma potential $\eta_{\infty}$ as a function of the charge source ratio $J_{i e}$ as given by Eq. (15) with $\delta=20, \gamma=3$, and for different temperature ratios: $\tau=0.1$ (top, black), $\tau=1$ (middle, blue), and $\tau=3$ (bottom, red). Different density ratios are considered: $\tilde{n}_{l} / \tilde{n}_{r}=0.66$ (left, dashed), $\tilde{n}_{l} / \tilde{n}_{r}=1$ (middle, solid), and $\tilde{n}_{l} / \tilde{n}_{r}=1.25$ (right, dashed-dotted).

$$
J_{t}=\frac{1}{\sqrt{\mu}} \frac{\tilde{n}_{l}}{\tilde{n}_{r}} \sqrt{\frac{1+\gamma \tau \tilde{n}_{l}^{\gamma-1}}{\tau+\gamma \tilde{n}_{r}^{\gamma-1}}} .
$$

A weak dependence of $J_{t}$ on $\tau$ is found, as displayed in Fig. 3. Thus, $\sqrt{\mu} J_{t}$ mainly depends on the ratio of sheath edge densities. Figure 3 shows the dependence of $J_{t}$ on $\tilde{n}_{l} / \tilde{n}_{r}$. We can make a rough estimate of the expected density ratio $\tilde{n}_{l} / \tilde{n}_{r}$. In the collisionless, isothermal limit, and neglecting sources and inertia, the density drop in the presheath is given by the Boltzmann factor. Also, in order to accelerate ions to sound speed (left presheath) and electrons to thermal speed (right presheath), both presheath potential drops are expected to be approximately equal to $T_{e \infty} / 2$. As a consequence, we expect $\tilde{n}_{l} / \tilde{n}_{r} \simeq 1$ for $\tau \sim 1$, implying that $J_{t} \simeq 1 / \sqrt{\mu}$.

On the other hand, the sharpness of the transition is strongly dependent on the temperature ratio $\tau$. In fact, as one can see in Fig. 3, the smaller the value of $\tau$, the steeper is the approach of $\eta_{\infty}$ to $\delta$ when $J_{i e}>J_{t}$. To quantify this, we consider the limit of Eq. (15) when $\eta_{\infty} \rightarrow \delta$, which is

$$
\lim _{\eta_{\infty} \rightarrow \delta} J_{i e}=J_{t} \sqrt{\frac{\tau+\gamma \tilde{n}_{r}^{\gamma-1}}{\gamma \tilde{n}_{r}^{\gamma-1}}}+\sqrt{\frac{2 \tau}{\mu \pi \gamma \tilde{n}_{r}^{\gamma-1}}} .
$$

Equation (17) gives $J_{t}$ for $\tau=0$, therefore a very sharp transition, and is a monotonically increasing function of $\tau$. This explains why the potential transition is more abrupt for small values of $\tau$.

We finally remark that the function $J_{i e}\left(\eta_{\infty}\right)$ does not strongly depend on the value of $\gamma$, which is expected to lie in between $\gamma=5 / 3$ (three-dimensional flow) and $\gamma=3$ (onedimensional flow). Therefore, the value of the transition current mainly depends on $\mu$ and $\tilde{n}_{l} / \tilde{n}_{r}$, and the sharpness of the potential transition mainly depends on $\tau$.

\section{NUMERICAL SIMULATIONS}

In order to confirm the validity of the analytical results presented in Sec. III, we perform numerical simulations with the ODISEE (one-dimensional sheath edge explorer) code, ${ }^{15}$ a fully kinetic, electrostatic PIC code akin to previous simulations. ${ }^{16,17} \mathrm{We}$ simulate an one-dimensional plasma bound between two absorbing walls at $x=0$ and $x=L$, where $L$ is much larger than the sheath scale, $L \gg \lambda_{D}$. A source of ions and electrons maintains the plasma in steady-state. Sources are located in the central region $[L / 3,2 L / 3]$ in order to avoid an influence on the sheath dynamics and are taken to be spatially uniform in this interval. In velocity space, ions, and electrons are injected according to a Maxwellian distribution with zero average velocity and temperatures $T_{i, s}$ and $T_{e, s}$, respectively. Notice that, as commonly observed in PIC simulations, ${ }^{18}$ the steady state bulk plasma temperatures, $T_{i \infty}$ and $T_{e \infty}$, are not necessarily equal to the corresponding source temperatures, therefore we cannot choose a priori the value of $\tau=T_{i \infty} / T_{e \infty}$. Electrons and ions undergo selfcollisions according to a Fokker-Planck collision operator, ${ }^{19}$ with a mean free path $\lambda_{m f p}$ smaller than the system size but much larger than the sheath scale, i.e., $L>\lambda_{m f} \gg \lambda_{D}$. As a

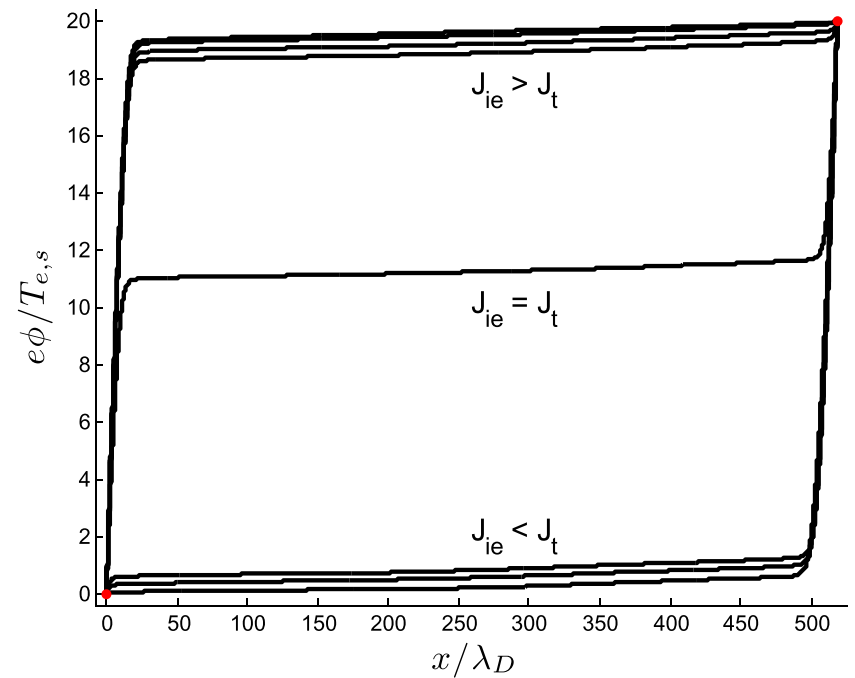

FIG. 4. Time averaged profiles of the plasma potential for $e\left(\phi_{w}^{r}-\phi_{w}^{l}\right) / T_{e, s}=20, \tau_{s}=1$, and for different values of $J_{i e}$. Top curves are for $\sqrt{\mu} J_{i e}=1.05,1.1,1.3,1.5$. Middle curve is for $\sqrt{\mu} J_{i e}=1$. Bottom curves are for $\sqrt{\mu} J_{i e}=0.95,0.9,0.7$. 


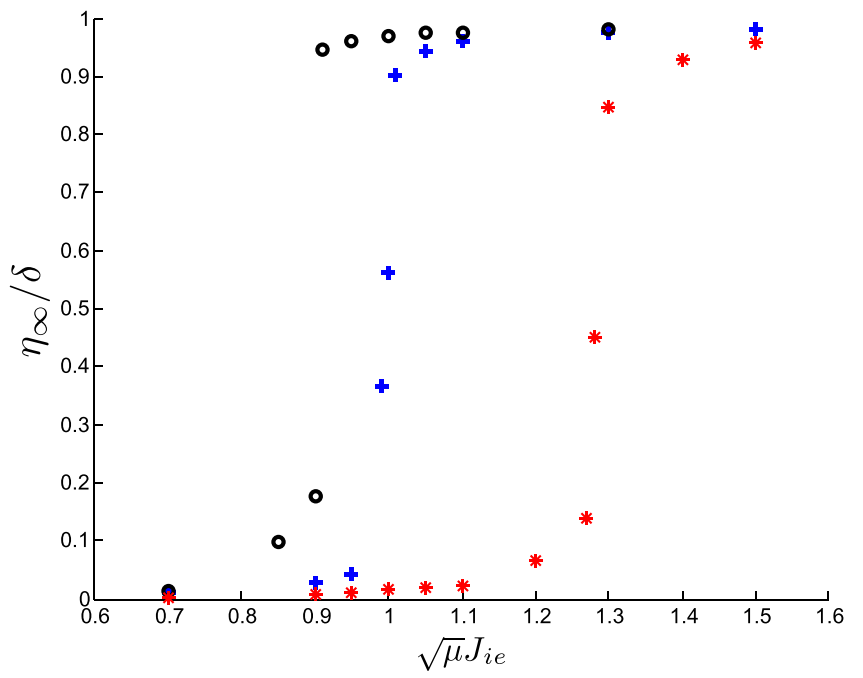

FIG. 5. Steady state plasma potential as a function of $J_{i e}$, for $\tau_{s}=0.5$ (black circles), $\tau_{s}=1$ (blue crosses), and $\tau_{s}=3$ (red stars). For all simulations, $e\left(\phi_{w}^{r}-\phi_{w}^{l}\right) / T_{e, s}=20$.

consequence, particles present a thermalized distribution function far from the walls, while the sheath remains essentially collisionless. Finally, Poisson's equation is solved by imposing the potential at the two boundaries, $\phi(0)=\phi_{w}^{l}$ and $\phi(L)=\phi_{w}^{r}$, such that $e\left(\phi_{w}^{r}-\phi_{w}^{l}\right) / T_{e, s} \gg 1$. The mass ratio is set to $\mu=100$. Sheath currents can be driven by varying the relative intensity of the ion and electron sources, therefore varying $J_{i e}$.

Figure 4 shows time-averaged profiles of the plasma potential from simulations performed with bias $e\left(\phi_{w}^{r}\right.$ $\left.-\phi_{w}^{l}\right) / T_{e, s}=20$ and $\tau_{s}=T_{i, s} / T_{e, s}=1$. A number of simulations are performed for different values of $J_{i e}$ around the estimated transition current ratio, namely, $J_{t} \approx 1 / \sqrt{\mu}$ as $\tilde{n}_{l} \simeq \tilde{n}_{r}$. Clearly, an abrupt transition in the plasma potential is observed when the charge source ratio $J_{i e}$ is slightly varied around $J_{i e}=1 / \sqrt{\mu}$, and variations of less than $5 \%$ around this value are enough to bring the potential of the plasma

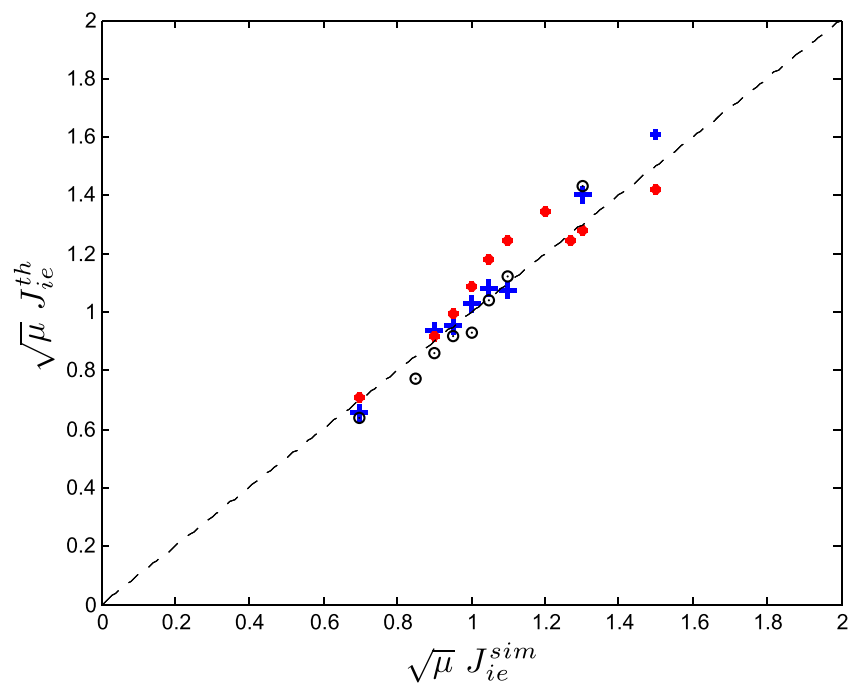

FIG. 6. Comparison between the current ratio $J_{i e}^{t h}$ predicted by Eq. (15) with $\gamma=3$ and the corresponding current ratio $J_{i e}^{\text {sim }}$ used as a numerical parameter in the simulation. Labels are the same as in Fig. 5. Dashed line indicates $J_{i e}^{\text {th }}=J_{i e}^{\text {sim }}$. bulk from one wall potential to the other wall potential. As discussed in Sec. III, this behavior is mainly due to the exponential dependence of the ion sheath electron current on the bulk plasma potential.

In Fig. 5, we show the bulk plasma potential as a function of $J_{i e}$ for different values of $\tau_{s}$. The presence of a sharp transition closely recalls the analytical results of Fig. 3. We remark that the comparison with the curves in Fig. 3 can only be qualitative, since a curve with constant $\tau_{s}$ does not exactly correspond to a curve with constant $\tau$.

In order to accurately verify the general analytical expression in Eq. (15), we proceed as follows. A set of simulations is performed where $\tau_{s}$ and $J_{i e}$ are varied. Each pair of parameters $\left(\tau_{s}, J_{i e}\right)$ produces a certain steady state, from which $\phi(L / 2), \tilde{n}_{l}, \tilde{n}_{r}, T_{i \infty}$, and $T_{e \infty}$ are extracted. One can then obtain the following parameters: $\tau=T_{i \infty} / T_{e \infty}, \eta_{\infty}$ $=e\left(\phi(L / 2)-\phi_{w}^{l}\right) / T_{e \infty}$, and $\delta=e\left(\phi_{w}^{l}-\phi_{w}^{r}\right) / T_{e \infty}$. Finally, the theoretical prediction for $J_{i e}$ is computed using Eq. (15) and compared with the corresponding simulation parameter. This exercise is carried out for different values of $\tau_{s}$ and $J_{i e}$. Figure 6 shows the results of this comparison, which confirms the validity of Eq. (15).
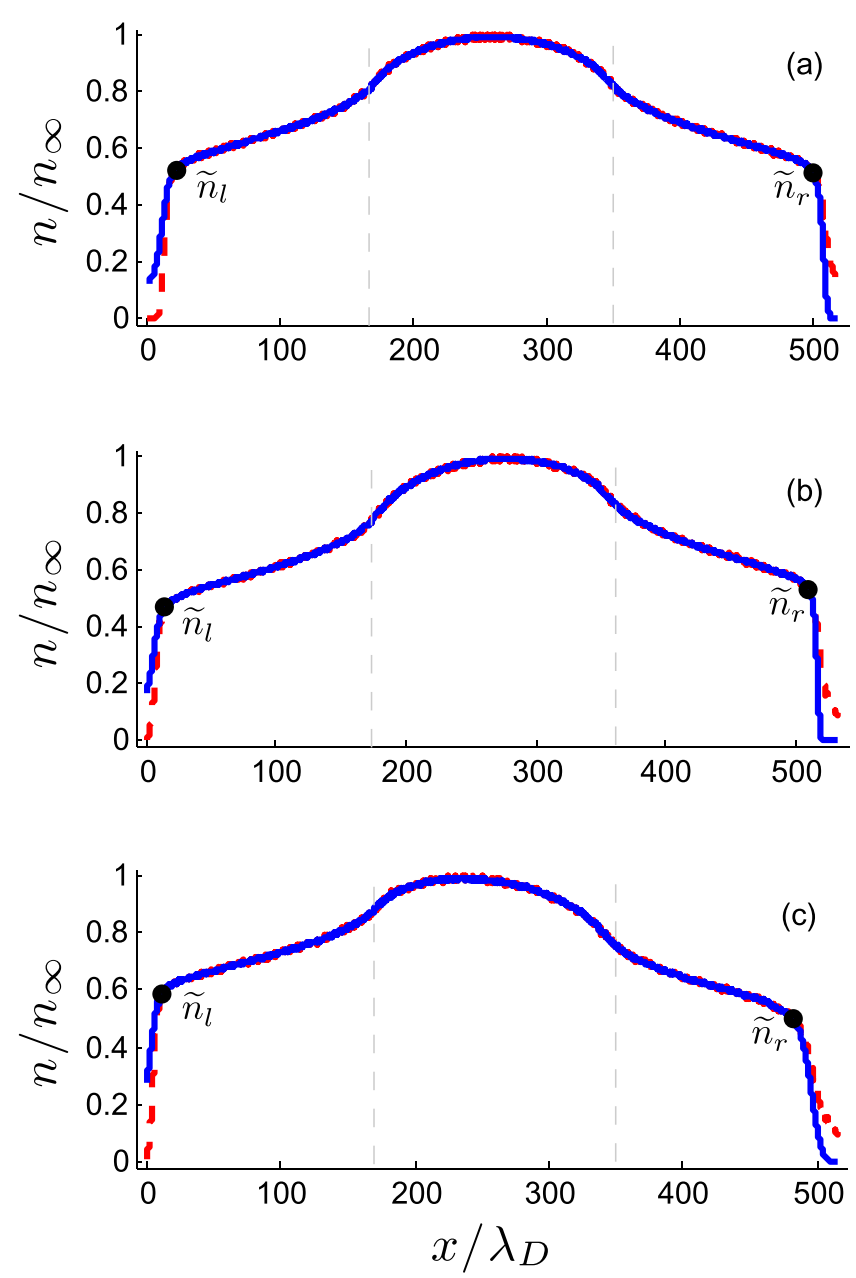

FIG. 7. Time averaged profiles of the ion density (solid, blue) and the electron density (dashed, red) for $e\left(\phi_{w}^{r}-\phi_{w}^{l}\right) / T_{e, s}=20$ and $J_{i e} \simeq J_{t}$. (a) $\tau_{s}=1$, (b) $\tau_{s}=0.5$, and (c) $\tau_{s}=3$. Indicated are the normalized sheath edge densities. The source is located between the two vertical dashed lines. 
We now discuss the dependence of the transition current ratio $J_{t}$ on the sheath edge densities $\tilde{n}_{l}$ and $\tilde{n}_{r}$. According to Eq. (16), the value of $J_{t}$ mainly depends on the mass ratio $\mu$ and the density ratio $\tilde{n}_{l} / \tilde{n}_{r}$. In simulations with $\tau_{s}=1 \simeq \tau$, the sheath edge densities are about the same on both sides, as shown in Fig. 7(a). This explains why, in the case displayed in Fig. 5, the potential transition occurs at $J_{i e} \simeq 1 / \sqrt{\mu}$. In simulations with $\tau_{s}=0.5$, however, the sheath edge densities are not the same on both sides, $\tilde{n}_{l} \lesssim \tilde{n}_{r}$ (see Fig. 7(b)). Therefore, the potential transition occurs at smaller values of $J_{i e}$, as expected from Eq. (16). An opposite trend is observed in simulations performed with $\tau_{s}=3$, namely $\tilde{n}_{l} \gtrsim \tilde{n}_{r}$ (see Fig. 7(c)). As one can observe in Fig. 7, the density ratios $\tilde{n}_{l} / \tilde{n}_{r}$ are always approximately equal to 1 . In particular, $\tilde{n}_{l} / \tilde{n}_{r} \simeq 0.9$ for $\tau_{s}=0.5$, and $\tilde{n}_{l} / \tilde{n}_{r} \simeq 1.2$ for $\tau_{s}=3$. In Fig. 7, one can also note that the left sheath is positively charged with $n_{i}>n_{e}$ (ion sheath) and the right sheath is negatively charged with $n_{e}>n_{i}$ (electron sheath).

We finally remark that, in the limit of $\tau=0$, simulations show an unstable behavior of the bulk plasma potential, which oscillates between $\eta_{\infty} \simeq 0$ and $\eta_{\infty} \simeq \delta$. These oscillations may be due to kinetic instabilities such as the twobeam instability. ${ }^{20}$

\section{DISCUSSION AND CONCLUSION}

When a bias is locally applied with a probe or at the wall confining a plasma and if the bias is strongly positive with respect to the potential of the vessel wall, the resulting plasma potential has a value that is usually between the two surface potentials. Therefore, an ion sheath forms at one wall and an electron sheath forms at the other wall. In order to analyse such plasmas, the ion and electron velocities at the entrance of both ion and electron sheaths have been rigorously derived. These results have been used to study the case of an one-dimensional, steady-state plasma bound between an ion sheath and an electron sheath. An analytical expression has been derived, relating the bulk plasma potential, $\eta_{\infty}$, to the ratio of currents drawn at the biased walls, $J_{i e}$, through a few parameters, mainly the bias amplitude $\delta$, the mass ratio $\mu$, the temperature ratio $\tau$, and the normalized sheath edge densities at both sides, $\tilde{n}_{l}$ and $\tilde{n}_{r}$. This analytical expression has been verified with PIC simulations carried out with the ODISEE code.

The bulk potential $\eta_{\infty}$ is found to be close to either of the two wall potentials for most values of $J_{i e}$ and shows an abrupt transition between these two potentials around a value $J_{t} \sim 1 / \sqrt{\mu}$. While this transition current ratio $J_{t}$ mainly depends on $\mu$ and $\tilde{n}_{l} / \tilde{n}_{r}$, the shape of the curve $\eta_{\infty}\left(J_{i e}\right)$ is strongly modulated by $\tau$. A setup that would allow the experimental determination of the curve $\eta_{\infty}\left(J_{i e}\right)$ could, therefore, provide a measure of the ion to electron temperature ratio in the plasma and constrain the values of $\mu$.

The analysis presented in this paper provides a tool to interpret the results of experiments where a part of the wall is positively biased. Our results indicate that, in most cases, the plasma potential has to be close to either of the wall potentials, depending on whether $J_{i e}<J_{t}$ or $J_{i e}>J_{t}$. In the particular situation of $J_{i e} \simeq J_{t}$, we expect that the plasma fluctuates between the two wall potentials due to possible slight variations of the sheath currents around $J_{t}$.

\section{ACKNOWLEDGMENTS}

We acknowledge many useful discussions with F. Avino, A. Bovet, A. Fasoli, I. Furno, K. Gustafson, and T.-M. Tran. We also acknowledge financial support by the Fonds National Suisse de la Recherche Scientifique.

${ }^{1}$ K.-U. Riemann, J. Phys. D. Appl. Phys. 24, 493 (1991).

${ }^{2}$ I. H. Hutchinson, Principles of Plasma Diagnostics (Cambridge University Press, Cambridge, 2002).

${ }^{3}$ K. M. Frederick-Frost and K. A. Lynch, Phys. Plasmas 14, 123503 (2007).

${ }^{4}$ E. Ahedo, Plasma Phys. Controlled Fusion 53, 124037 (2011).

${ }^{5}$ A. Douglass, V. Land, K. Qiao, L. Matthews, and T. Hyde, Phys. Plasmas 19, 013707 (2012).

${ }^{6}$ T. A. Carter and J. E. Maggs, Phys. Plasmas 16, 012304 (2009).

${ }^{7}$ D. L. Toufen, Z. O. Guimares-Filho, I. L. Caldas, F. A. Marcus, and K. W. Gentle, Phys. Plasmas 19, 012307 (2012).

${ }^{8}$ B. Li, B. N. Rogers, P. Ricci, K. W. Gentle, and A. Bhattacharjee, Phys. Rev. E 83, 056406 (2011).

${ }^{9}$ A. G. Lynn, M. Gilmore, C. Watts, J. Herrea, R. Kelly et al., Rev. Sci. Instrum. 80, 103501 (2009).

${ }^{10}$ C. Theiler, I. Furno, J. Loizu, and A. Fasoli, Phys. Rev. Lett. 108, 065005 (2012).

${ }^{11}$ D. D. Ryutov, P. Helander, and R. H. Cohen, Plasma Phys. Controlled Fusion 43, 1399 (2001).

${ }^{12}$ S. J. Zweben et al., Plasma Phys. Controlled Fusion 51, 105012 (2009).

${ }^{13}$ S. D. Baalrud, N. Hershkowitz, and B. Longmier, Phys. Plasmas 14, 042109 (2007)

${ }^{14}$ S. Kuhn, Contrib. Plasma Phys. 34(4), 495-538 (1994).

${ }^{15}$ J. Loizu, P. Ricci, and C. Theiler, Phys. Rev. E 83, 016406 (2011)

${ }^{16}$ J. R. Procassini et al., Phys. Fluids B 2, 12 (1990).

${ }^{17}$ J. R. Procassini et al., Phys. Fluids B 3, 8 (1991).

${ }^{18} \mathrm{P}$. Stangeby, The Plasma Boundary of Magnetic Fusion Devices (IOP, Bristol, 2000).

${ }^{19}$ T. Takizuka, J. Comput. Phys. 25, 205-219 (1977).

${ }^{20}$ G. G. Comisar, J. Appl. Phys. 38, 905-907 (1967). 